\title{
Ultracold antiprotons by indirect laser cooling
}

\author{
A. Kellerbauer · C. Canali · A. Fischer · U. Warring
}

Published online: 29 August 2009

(C) The Author(s) 2009. This article is published with open access at Springerlink.com

\begin{abstract}
The performance of proposed antihydrogen spectroscopy or gravity experiments will crucially depend on the temperature of the initial antihydrogen sample. Measurements by ATRAP and ATHENA have shown that antihydrogen produced with the nested-trap technique is much hotter than the temperature of the surrounding trap. Therefore, novel schemes for antihydrogen recombination as well as for the pre-cooling of antiprotons are being considered. We are investigating a possible antiproton cooling technique based on the laser cooling of negative osmium ions. If demonstrated to be successful, it will allow the sympathetic cooling of antiprotons - or any negatively charged particles—-to microkelvin temperatures. As a first milestone toward the laser cooling of negative ions, we have performed collinear laser spectroscopy on negative osmium and determined the transition frequency and the cross-section of the relevant bound-bound electric-dipole transition.
\end{abstract}

Keywords Atomic anion • Osmium • Collinear spectroscopy • Ion cooling

PACS $32.80 . \mathrm{Gc} \cdot 32.30 . \mathrm{Bv} \cdot 32.70 . \mathrm{Cs} \cdot 37.10 . \mathrm{Rs}$

\section{Toward ultracold antihydrogen}

The first creation of atomic antihydrogen $(\overline{\mathrm{H}})$ at CERN's low-energy antiproton ring (LEAR) in 1995 [1] has fueled an immense interest in antimatter studies. Since then, several groups of scientists at the CERN antiproton decelerator (AD) have

This work was supported by the German Research Foundation (DFG) by an Emmy Noether grant under Contract No. KE1369/1-1.

A. Kellerbauer $(\bowtie) \cdot$ C. Canali · A. Fischer · U. Warring Max Planck Institute for Nuclear Physics, P.O. Box 103980, 69029 Heidelberg, Germany e-mail: a.kellerbauer@mpi-hd.mpg.de 
been attempting to create the largest possible numbers of anti-atoms at the lowest achievable temperatures. The ultimate aim is to eventually perform tests of CPT symmetry (invariance under the simultaneous operations of charge conjugation, parity and time reversal) and the weak equivalence principle with $\overline{\mathrm{H}}$ [2]. The temperature of the antihydrogen at the moment of its formation is of the utmost importance because it determines the fraction of neutral anti-atoms that can be confined in a radial magnetic multipole trap [3] or, alternatively, the quality of an antihydrogen beam accelerated out of the recombination region.

The ATHENA and ATRAP experiments were the first, in 2002, to successfully synthesize $\overline{\mathrm{H}}[4,5]$ from antiprotons pre-cooled with an electron plasma [6] and positrons, both stored in Penning traps. The highest recombination rates and absolute numbers of $\overline{\mathrm{H}}$ atoms [7] were achieved using the so-called nested-well technique [8], which allows the simultaneous confinement of negative antiprotons and positively charged positrons. Measurements of the $\overline{\mathrm{H}}$ velocity distribution [9] and of the spatial distribution of annihilation events [10] have shown, however, that this antihydrogen recombination method produces anti-atoms at several orders of magnitude higher temperatures than that of the surrounding environment ( $4 \mathrm{~K}$ to $15 \mathrm{~K})$. Antihydrogen production based on a charge exchange reaction between highly excited positronium and antiprotons at rest $[11,12]$ should create $\overline{\mathrm{H}}$ that essentially has the temperature of the antiprotons before recombination. A variation of this scheme [13] has recently been demonstrated [14], and it will also be employed in the proposed AEGIS antimatter gravity experiment [15]. Therefore, a mechanism is required by which antiprotons can be cooled to cryogenic temperatures well below that of the surrounding environment.

The well-established technique of laser cooling, first applied to atomic ions [16] and neutral atoms [17] several decades ago, is unfortunately not applicable to antiprotons, which are subatomic particles without an atomic structure. They can, however, be cooled by collisions with other particles, a process that is called sympathetic cooling. As the antiprotons cool, their temperature asymptotically approaches that of the cooling medium, which is at best in thermal equilibrium with the cryogenic environment. This restriction can be overcome if the ions which are used for sympathetic cooling are actively cooled. Atomic ions that are confined in the same trap region as the antiprotons could be laser-cooled and would then successively remove thermal energy from the $\bar{p}$ cloud until the Doppler temperature is reached [18]. However, since antiprotons are negatively charged, only negative ions can be employed, because positive ions are attracted to the $\bar{p}$ by the Coulomb force and would rapidly annihilate with them. In contrast with the successful laser cooling of positive ions and neutral atoms mentioned above, the laser cooling of negative ions has never been attempted. This is because until recently, no negative ion was known which exhibits a bound-bound transition that is appropriate for laser cooling.

\section{Laser cooling of negative ions}

The structure of negative atomic ions is fundamentally different from that of neutral atoms or positive ions because of the nature of the potential experienced by the 
Fig. 1 (Color online.) Energy level diagram of the negative osmium ion. The red arrow indicates the relevant transition for laser cooling

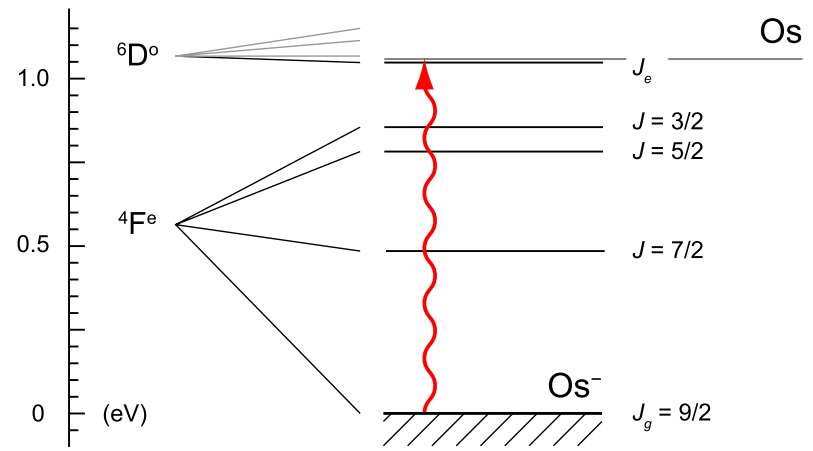

valence electron [19]. Classically, negative ions should not exist, as it is not energetically favorable for a negatively charged electron to attach itself to a neutral core. Nevertheless, most elements form negative ions. They are created by polarization of the neutral atom and are stable due to quantum-mechanical correlation effects. Their binding energy, the energy gained when all $Z+1$ electrons adjust their wavefunctions in accordance with the Pauli exclusion principle and electrostatic repulsion, is typically about an order of magnitude smaller than the binding energies of atoms or positive ions. The potential is both shallow and short-ranged; therefore, only a limited number of bound states (if any) exists.

The number of negative ions which form bound excited states is even smaller [20]. Most of these states are sub-levels of the same configuration and hence have the same parity as the ground state. Due to the well-known selection rules, electricdipole transitions cannot occur between same-parity states. Such transitions are, however, of particular interest for spectroscopic investigations. Moreover, they could in principle be used to laser-cool the negative ion. Opposite-parity bound states have been predicted for the anions of a number of elements. While some of these candidates have not yet been investigated experimentally, the existence of such states in lanthanum and cesium has already been ruled out [21, 22].

Recently, a comparatively strong resonant transition just below the photodetachment threshold was discovered in the negative osmium ion and investigated by infrared laser photodetachment spectroscopy [23]. In this study of $\mathrm{Os}^{-}$, the transition frequency (wavelength $\lambda \approx 1162.7 \mathrm{~nm}$ ) was determined with an uncertainty of $\approx 5 \mathrm{GHz}$. It was found that the bound excited state is very weakly bound (binding energy $\approx 11.5 \mathrm{meV}$ ) and that its Einstein coefficient is $A \approx 10^{4}$. Figure 1 shows the resulting energy level diagram, taking into account theoretical calculations on the ground state configuration [24]. The narrow linewidth means that the Doppler temperature achievable by laser cooling is $T_{D} \approx 0.24 \mu \mathrm{K}$, four orders of magnitude lower than that of (anti-)hydrogen when using the Lyman- $\alpha$ transition [25]. Based on these experimental data, the aforementioned theoretical study [18] established that the laser cooling of $\mathrm{Os}^{-}$should be technically feasible. Many aspects of the technique, however, depend on the cross-section of the cooling transition as well as the configuration of the bound state, necessitating a more detailed spectroscopic investigation of $\mathrm{Os}^{-}$. 


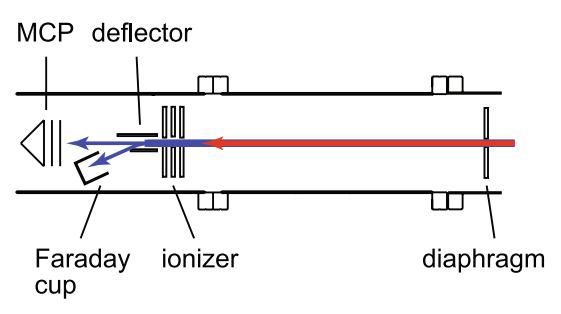

(a)

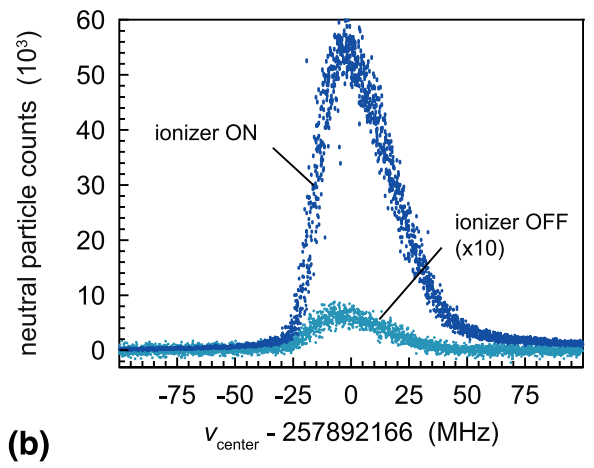

Fig. 2 (Color online.) a Sketch of the spectroscopy section of the UNIC apparatus showing entrance and exit diaphragms, ionizer, deflector, MCP detector and Faraday cup. b Typical resonance of the bound-bound electric-dipole transition, at an ion beam energy of $5 \mathrm{keV}$ [28]. The figure illustrates the efficiency gain of about two orders of magnitude due to the field detachment process

\section{High-resolution laser spectroscopy on $\mathrm{Os}^{-}$}

The UNIC apparatus (Ultracold Negative Ions by indirect laser Cooling) at the Max Planck Institute for Nuclear Physics in Heidelberg consists of a negative ion source, a mass separator, a Penning trap contained in the magnetic field of a superconducting magnet, and a laser system. Negative Os ions are produced in a Middletontype sputter ion source [26]. They are extracted from the source, accelerated to $E=2.5 \ldots 6.5 \mathrm{keV}$ by electrostatic potentials and mass-separated by a large dipole magnet with a resolving power of $\approx 180$, resulting in a 50-nA beam which consists of more than $90 \%{ }^{192} \mathrm{Os}^{-}$. The Penning trap (diameter $32 \mathrm{~mm}$ ) consists of eleven cylindrical segments whose lengths and potentials were chosen such as to minimize the deviation of the electric field from an ideal 3-d quadrupole configuration [27]. The ring electrode contains radial orifices (diameter $4.5 \mathrm{~mm}$ ) by which ions confined in the trap can be illuminated with laser light. The laser is a commercial optical parametric oscillator (OPO) system custom-built for this application. When pumped at a power of $2 \mathrm{~W}$, the OPO produces close to $200 \mathrm{~mW}$ of output near $1162.7 \mathrm{~nm}$ with a bandwidth smaller than $5 \mathrm{MHz}$. The wavelength is recorded and regulated with a wavemeter, which in turn is calibrated by a stabilized diode laser.

For the purpose of the measurements presented here, a spectroscopy section was added to the apparatus, in which collinear laser spectroscopy can be carried out on the ion beam. A sketch of this section is shown in Fig. 2. It consists of entrance and exit diaphragms (diameter $7.5 \mathrm{~mm}$, length of interaction region $520 \mathrm{~mm}$ ), an ionizer, a deflector, a micro-channel-plate detector (MCP) and a Faraday cup. The ion beam and the laser beam are collinearly superimposed over the length of the entire interaction region. In this way, velocity bunching reduces the Doppler width of the resonance by up to a factor of 10 for our experimental conditions (ion temperature $\approx 1500 \mathrm{~K}$, beam energy $5 \mathrm{keV}$ ). In the ionizer, the beam passes through three parallel meshes placed in a plane perpendicular to the beam. A high voltage of up to $6.5 \mathrm{kV}$ applied to the central mesh gives rise to a longitudinal electric field of up to $2 \times 10^{6} \mathrm{~V} \mathrm{~m}^{-1}$. A deflector leads the ions into a Faraday cup, whereas the MCP counts neutralized particles which are not deflected. 
Fig. 3 (Color online.) Blue-shifted resonance frequencies as a function of the ion beam energy [28]. The solid line is the result of the fit for the Doppler shift, its extrapolation to zero beam energy is shown in the inset. The lower pane shows the residuals of the fit

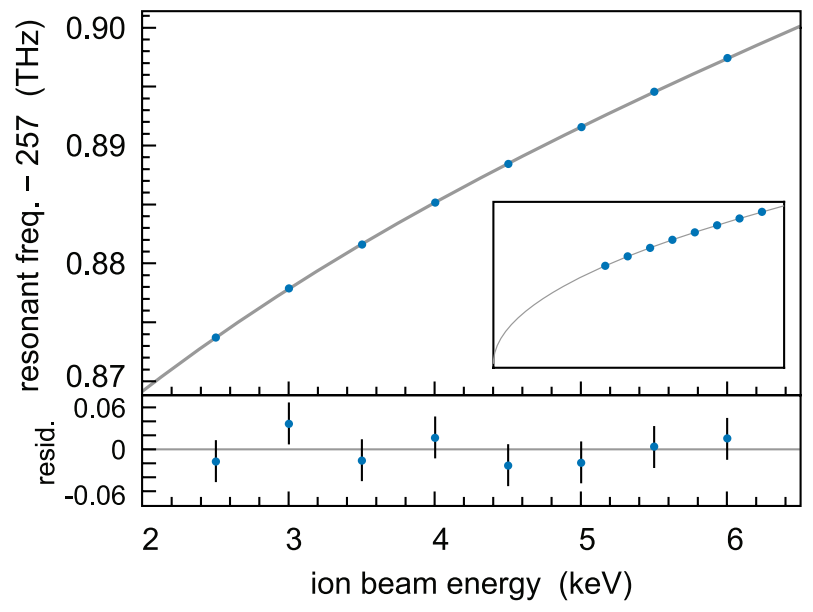

Previous investigations of excited states in negative ions have relied on photodetachment by absorption of an additional photon into the excited state. In our setup, ions which have been excited to the $J_{e}$ state in the interaction region are neutralized by the strong electric field in the ionizer. Of course, photodetachment nevertheless occurs and contributes to the total neutralization rate. All neutral atoms are detected by the MCP placed in the forward direction. A typical excitation resonance is shown in Fig. 2b, along with the corresponding resonance obtained without the ionizing potential. The difference in signal intensities illustrates the dramatic enhancement due to the field detachment. The width of the (mainly Gaussian) resonance, $\Gamma_{\text {res }} \approx 45 \mathrm{MHz}$, is dominated by the Doppler width; its slight asymmetry is due to a corresponding asymmetry in the velocity distribution of the ions.

In collinear laser spectroscopy, the measured transition frequency is blue-shifted because of the Doppler effect. While the transition frequency in the ion's rest frame can be deduced from a single measurement at a well-known ion beam energy, a more precise value is obtained by performing a number of measurements at different beam energies and fitting the data points to the well-known function for the Doppler shift. Furthermore, a possible systematic shift in the beam energy can be accounted for by including it as a parameter of the fit. The result of these measurements and the corresponding fit are shown in Fig. 3 [28]. From the fit, a transition frequency of $v_{0}=257.831190(35) \mathrm{THz}$ was obtained, corresponding to a wavelength of 1162.74706(16) nm. This is in good agreement with the prior measurement [23], but more than two orders of magnitude more precise. The fit yielded an average beam energy error of $0.4(5) \mathrm{eV}$. To our knowledge, this transition frequency measurement constitutes the most precise determination of any feature in an atomic anion.

The resonant cross-section can be determined by considering the time evolution of the ground and excited state populations in the beam as well as the number of neutralized atoms. A set of three differential rate equations for these populations in the region of overlapping beams can be solved analytically [29]. The total number of neutralized particles is obtained by numerically integrating the expressions for the number of excited and detached ions over time and the radial extent of the overlapping beams. Assuming constant overlap of the ion and laser beam, it is only 
Fig. 4 (Color online.) Number of detected atoms as a function of the laser intensity, recorded in resonance and for a fixed beam energy [28]. The solid line is a fit to the data according to the rate equation model

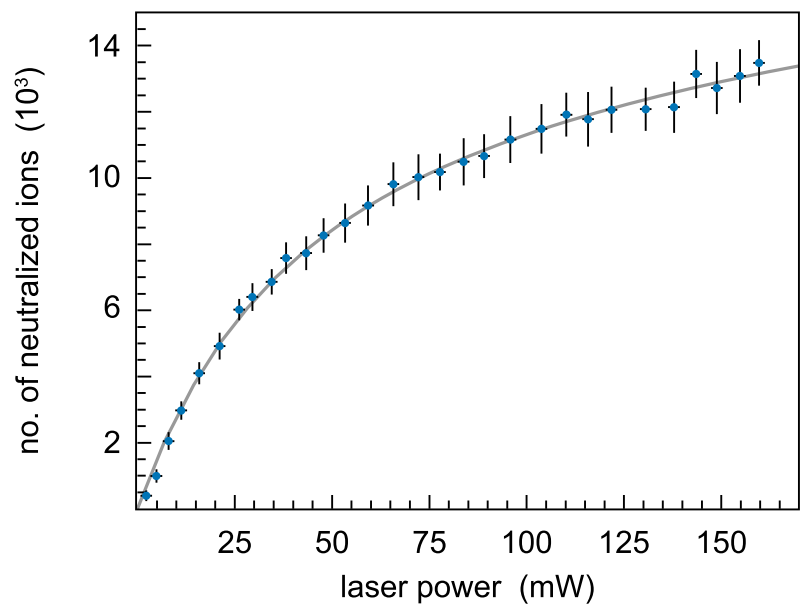

a function of the laser power and has four independent parameters: The resonantexcitation and detachment cross-sections $\sigma_{0}$ and $\sigma_{d}$, the number of ions within the overlap region $N_{0}$, and the mean lifetime $\tau$ of the excited state with respect to spontaneous emission to the ground state. Neglecting decay to intermediate states, the latter is related to the cross-section via $\tau=c^{2} /\left(4 \pi^{2} \sigma_{0} v_{0}^{2} \Gamma_{\text {res }}\right)$, but was nevertheless treated as an independent parameter of the function.

Figure 4 shows a measurement of the total number of neutralized ions detected on the MCP as a function of the laser power at an ion beam energy of $2.5 \mathrm{keV}$, with a fit to the rate equation model as described above [28]. Deviating somewhat from the initial assumption of constant overlap between the beams, ion-optical simulations have shown that the ion beam diverges by about $1.5^{\circ}$ in the interaction region, effectively reducing the overlap volume by about $30 \%$. Taking this effect into account, we obtained a resonant cross-section of $\sigma_{0}=2.5(7) \times 10^{-15} \mathrm{~cm}^{2}$ from a total of 10 measurements for various beam energies. Due to the fairly long mean lifetime $\tau$ of the excited state compared with the transit time in the interaction region, the corresponding fit parameter is not well constrained. It can, however, be determined indirectly from the cross-section via the relation given in the preceding paragraph. In this way, we obtained a value of $\tau=3(1) \mathrm{ms}$. The measured observed cross-section yields an Einstein coefficient of $A \approx 330 \mathrm{~s}^{-1}$, compared with $A \approx 10^{4} \mathrm{~s}^{-1}$ found in Ref. [23], confirming that the resonance is due to a weak, spin-forbidden electricdipole transition.

Because of the small Einstein $A$ coefficient, the characteristics of the laser cooling of $\mathrm{Os}^{-}$, such as the Doppler temperature as well as the appropriate detuning and required laser power will be determined by the laser bandwidth. Even in saturation, the cooling rate cannot exceed $A /(2 \pi) \approx 50 \mathrm{~Hz}$, and cooling times from $80 \mathrm{~K}$ to the Doppler temperature would be prohibitively long. Therefore, it may be necessary to pre-cool the ions to liquid-helium temperature or even below by sympathetic cooling with electrons. The laser cooling from $4 \mathrm{~K}$ to the Doppler temperature would then take roughly 5 minutes. In summary, we have performed high-resolution collinear laser spectroscopy on the negative osmium ion as a first milestone toward the laser cooling of $\mathrm{Os}^{-}$. As a result, we have determined the transition frequency of the 
bound-bound electric-dipole transition to a relative precision of $10^{-7}$ and carried out a measurement of the resonant cross-section. Laser cooling of $\mathrm{Os}^{-}$should be feasible, but will likely require the pre-cooling of the negative ions to liquid-He temperature.

Acknowledgements We acknowledge the support of the MPIK workshop and accelerator groups, in particular by M. König, V. Mallinger and M. Beckmann, as well as the assistance of the group of S. Jochim for the wavemeter calibration.

Open Access This article is distributed under the terms of the Creative Commons Attribution Noncommercial License which permits any noncommercial use, distribution, and reproduction in any medium, provided the original author(s) and source are credited.

\section{References}

1. Baur, G., et al.: Phys. Lett. B 368, 251 (1996). doi:10.1016/0370-2693(96)00005-6

2. Holzscheiter, M.H., et al.: Phys. Rep. 402, 1 (2004). doi:10.1016/j.physrep.2004.08.002

3. Pritchard, D.E.: Phys. Rev. Lett. 51, 1336 (1983). doi:10.1103/PhysRevLett.51.1336

4. Amoretti, M., et al.: Nature 419, 456 (2002). doi:10.1038/nature01096

5. Gabrielse, G., et al.: Phys. Rev. Lett. 89, 213401 (2002). doi:10.1103/PhysRevLett.89.213401

6. Gabrielse, G., et al.: Phys. Rev. Lett. 63, 1360 (1989). doi:10.1103/PhysRevLett.63.1360

7. Amoretti, M., et al.: Phys. Lett. B 578, 23 (2004). doi:10.1016/j.physletb.2003.10.062

8. Gabrielse, G., et al.: Phys. Lett. A 129, 38 (1988). doi:10.1016/0375-9601(88)90470-7

9. Gabrielse, G., et al.: Phys. Rev. Lett. 93, 073401 (2004). doi:10.1103/PhysRevLett.93.073401

10. Madsen, N., et al.: Phys. Rev. Lett. 94, 033403 (2005). doi:10.1103/PhysRevLett.94.033403

11. Humberston, J.W., et al.: J. Phys. B 20, L25 (1987). doi:10.1088/0022-3700/20/1/005

12. Charlton, M.: Phys. Lett. A 143, 143 (1990). doi:10.1016/0375-9601(90)90665-B

13. Hessels, E.A., et al.: Phys. Rev. A 57, 1668 (1998). doi:10.1103/PhysRevA.57.1668

14. Storry, C.H., et al.: Phys. Rev. Lett. 93, 263401 (2004). doi:10.1103/PhysRevLett.93.263401

15. Drobychev, G., et al.: (AEGIS Proto-Collaboration), CERN-SPSC-2007-017 (2007). http:// cdsweb.cern.ch/record/1037532

16. Wineland, D. J., et al.: Phys. Rev. Lett. 40, 1639 (1978). doi:10.1103/PhysRevLett.40.1639

17. Chu, S., et al.: Phys. Rev. Lett. 55, 48 (1985). doi:10.1103/PhysRevLett.55.48

18. Larson, D. J., et al.: Phys. Rev. Lett. 57, 70 (1986). doi:10.1103/PhysRevLett.57.70

19. Pegg, D.J.: Rep. Prog. Phys. 67, 857 (2004). doi:10.1088/0034-4885/67/6/R02

20. Andersen, T., et al.: J. Phys. Chem. Ref. Data 28, 1511 (1999). doi:10.1063/1.556047

21. Covington, A.M., et al.: J. Phys. B 31, L855 (1998). doi:10.1088/0953-4075/31/20/002

22. Scheer, M., et al.: Phys. Rev. Lett. 80, 684 (1998). doi:10.1103/PhysRevLett.80.684

23. Bilodeau, R.C., Haugen, H.K.: Phys. Rev. Lett. 85, 534 (2000). doi:10.1103/PhysRevLett.85.534

24. Norquist, P.L., Beck, D.R.: Phys. Rev. A 61, 014501 (1999). doi:10.1103/PhysRevA.61.014501

25. Eikema, K.S.E., et al.: Phys. Rev. Lett. 86, 5679 (2001). doi:10.1103/PhysRevLett.86.5679

26. Middleton, R.: Nucl. Instrum. Methods 214, 139 (1983). doi:10.1016/0167-5087(83)90580-X

27. Raimbault-Hartmann, H., et al.: Nucl. Instrum. and Methods B 126, 378 (1997). doi:10.1016/ S0168-583X(96)01067-1

28. Warring, U., et al.: Phys. Rev. Lett. 102, 043001 (2009). doi:10.1103/PhysRevLett.102.043001

29. Loudon, R.: The Quantum Theory of Light, 3rd edn. Oxford, New York (2000) 